\title{
Computer system for identification of tool wear model in hot forging
}

\author{
Marek Wilkus ${ }^{1}$, Łukasz Rauch ${ }^{1}$, Zbigniew Gronostajski ${ }^{2}$, Sławomir Polak ${ }^{2}$, Maciej Pietrzyk ${ }^{1}$ \\ ${ }^{1}$ AGH University of Science and Technology, al. Mickiewicza 30, 30-059 Kraków, Poland \\ ${ }^{2}$ Wrocław University of Technology, ul. Łukasiewicza 5-7, 50-370 Wrocław, Poland
}

\begin{abstract}
The aim of the research was to create a methodology that will enable effective and reliable prediction of the tool wear. The idea of the hybrid model, which accounts for various mechanisms of tool material deterioration, is proposed in the paper. The mechanisms, which were considered, include abrasive wear, adhesive wear, thermal fatigue, mechanical fatigue, oxidation and plastic deformation. Individual models of various complexity were used for separate phenomena and strategy of combination of these models in one hybrid system was developed to account for the synergy of various mechanisms. The complex hybrid model was built on the basis of these individual models for various wear mechanisms. The individual models expanded from phenomenological ones for abrasive wear to multiscale methods for modelling micro cracks initiation and propagation utilizing virtual representations of granular microstructures. The latter have been intensively developed recently and they form potentially a powerful tool that allows modelling of thermal and mechanical fatigue, accounting explicitly for the tool material microstructure.
\end{abstract}

\section{Introduction}

In industrial hot forging processes, prediction of die wear is critical for optimization of costs and materials usage. Proper die wear model allows to design production process with minimal amount of material waste and minimal time losses due to technical stops [1]. Die wear is an effect of many physical phenomena, such as friction (abrasive wear), material sticking (adhesive wear), thermal and mechanical fatigue, oxidation of working layers of material, etc. $[3,2,4]$. These mechanisms depend on many factors, both related to process and material. Majority of wear models used today in engineering applications is focused on one mechanism, while effects of other are ignored. This approach makes the wear prediction less useful, especially if wear in large product series (industrial application) is calculated. In processes with different geometries and parameters, wear depends on different mechanisms to various degrees. Even in a single process, in different parts of die different wear mechanisms may be of primary effect. Dies for hot forging usually have surfaces enhanced some way (thermal processing, nitriding, etc.), so after enriched layer wears off, other wear mechanisms occur and different die material and surface parameters have to be used. In modelling of tool wear, it is not always easy to identify all coefficients in models. Beyond identification of a particular model, an importance of different wear mechanisms has to be analysed in various parts of the die.

The objectives of the paper have been formulated having above remarks in mind. Creating a methodology that will enable effective and reliable prediction of the tool wear was the main objective.

\section{Wear models}

Die wear is a phenomenon which depends on many factors such as friction, thermal conditions (temperature, heat transfer coefficients), contact time, number of forging cycles in the same die, chemical parameters of die or die geometry. Various wear mechanisms are usually affecting properties of the die. Accounting for the synergy of these mechanisms (a single wear mechanism changes effects of other mechanisms) is the main challenge in modelling. Archard model of abrasive wear [5] is the most commonly used tool wear model in engineering applications. Similar model was proposed by Shaw [6]. This model covers only wear related to friction. The wear depth $W$ is described in as:

$$
W=\int C \frac{\mu p v}{H V} d t
$$

where $C$ is a non-dimensional coefficient, $\mu$ is friction coefficient, $p$ is normal pressure, $v$ is sliding velocity and $H V$ is a hardness of a die material. In Authors previous work [7] it was shown that using this model sometimes requires changing of parameters after some wear threshold, which may be caused by changes of the die surface properties after enhanced layer (e.g. nitrided) wears off. To solve problem of large number of forgings, the model should be modified by introducing hardness dependent on various factors such as number of forgings, temperature, residual wear etc. [8]. 
Formula (1) can be also adapted to work with adhesive wear [9]. It was done by introducing hardness of the workpiece and adding exponential coefficients for friction, velocity and hardness parameters.

Simple models such as Paris equation [10] or more complex crack growth equations may be used for modelling of thermomechanical fatigue crack initiation and propagation. There is a variety of models available, from statistical or phenomenological ones, through formulas including deformation thresholds [11], to advanced formulas including deformation zones and crystallographic parameters changes [12]. Some of these formulas use parameters, which are difficult to obtain even from computer simulation, e.g. microstructural parameters of analysed part.

Another approach to modelling thermomechanical fatigue cracks is multi-scale analysis. In this approach statistically similar representative volume element (SSRVE) [13] of the die is analysed using Finite Element (FE) method (or e.g. cellular automata (CA) based on microstructure, meshless methods, etc.) or on a lower level (e.g. microstructure) returning material or crack properties to higher-level analysis. This approach may give more precise results, but it will require more computations (different parts of the die have different wear mechanisms) and must include changes of material properties (layer wear-off). Additionally, during subsequent forging cycles plastic deformation of crack as well as crack closure may occur, which strongly depends on materials ductility. Crack closing happens until some crack width is reached. After this, plastic deformation of workpiece occurs and fosters crack propagation. Thus, plastic deformation is another important wear mechanism of die itself. Plastic deformation increases by a small increment at each cycle, therefore, it is difficult to modelling. In many applications this mechanism has been simulated using model to suspected cumulative load [14], cycle simulation or steady-state simulation if possible [15]. Simulation of industrial cycle of forging is time and resource consuming. Thus, simple extrapolation-based models with geometry analysis have been used in the present work.

\section{Computer system idea}

To compute wear values, a hybrid computer system has been proposed, which combines numerical simulation of forging process with wear models. The schematic idea of this system is shown in figure 1. Parts of this system were created with user procedures implemented in commercial software, while other parts are standalone implementations. This approach allows further extension and quick testing of modifications. Execution of the system starts by specifying analysed problem (Problem component in figure 1). The problem consists of the following two parts:

- The numerical simulation of analysed process, prepared by using commercial tools.

- Information needed for wear modelling, e.g. initial die hardness used by abrasive wear module or chemical parameters for oxidation wear module.

The simulation is passed to the Compiler module which prepares the simulation and customizes it to compute the wear.

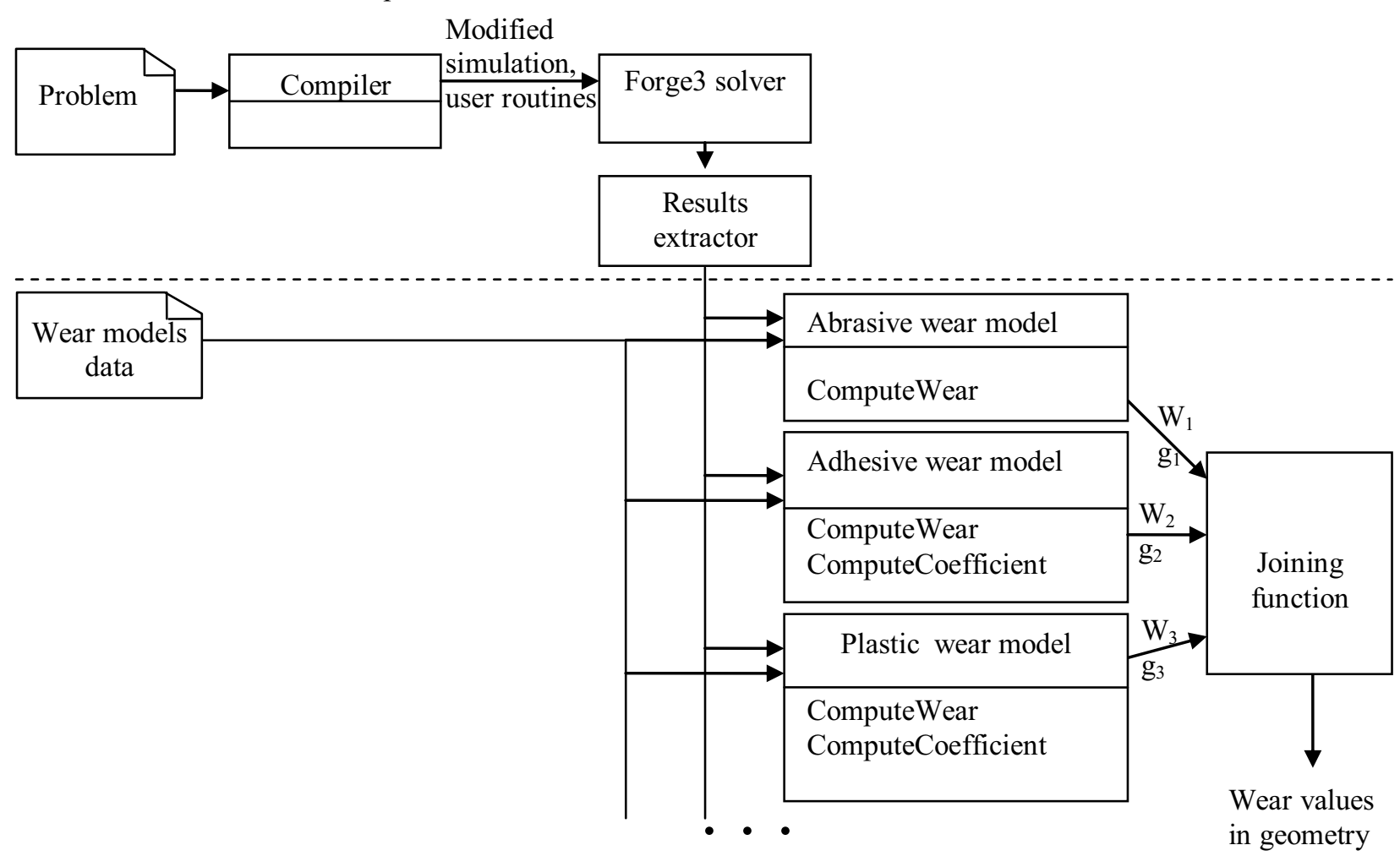

Figure 1. Schematic diagram of the computer system simulating hybrid wear model.

The most important parts of these customizations are: 
- Introduction of specific output variables definitions from solver for further computations (e.g. sliding distance).

- Copying modified solver's user routines if crack initiation or propagation is computed.

- Verification of a die mesh regarding surface elements and problems resulting from improper surface mesh; Optional surface remeshing to improve accuracy.

- Compiling simulation to a commercial software (Forge 3 component in figure 1); Optionally, generation of machine-specific files and start-up configurations.

- Execution of simulations.

After simulation finishes, post-processing routines are executed. Not only typical wear is computed, but also significance of different wear mechanisms must be resolved. Each post-processing module consists of procedure computing wear value and weight coefficient. Such models are getting information from data acquisition module. Separating solver from model using data acquisition and starting interface-like modules allows to develop the model with deeply modified solvers or different ones, as well as test multi-solver approach in future research. This architecture is commonly used in industry. The last stage of wear computation is applying joining function on wear models' results. This allows to obtain the final wear value.

\section{Numerical simulation}

Numerical simulation with Finite Element (FE) method was used to verify and evaluate wear models. There are many commercial FE packages for simulation of metal forming processes. All simulations in this work have been made using Forge 2011 package, which is widely used in engineering applications. This software uses Norton-Hoff constitutive law and has a large base of rheological models. It is also possible to add user routines, mainly for computation of scalar variables. To calculate wear, post-processing calculation has been used. The script-driven software for simulation automation has been implemented to form system based on a scheme shown in Figure 1. It allowed to prepare simulations with specially defined parameters as well as to perform multistage simulations with different variants of these parameters. Finally, the results are exported to compute wear using own models. Post-processing computations allow to find simple abrasive or other formula-defined wear equations, while e.g. special crack propagation models will have to be started with simulation. Evaluation of wear mechanisms begins with execution of a single simulation results of which are analysed to find importance of different wear mechanisms. Geometry properties are taken into account during analysis. For example, abrasive wear (computed from Archard model) depends on sliding distance, velocity and force. Contrary, the wear caused by plastic deformation of the die is in many cases caused by forces acting on specific parts of the die, prone to plastic deformation. The plastic effect of these forces is cumulative and it becomes visible when many forgings are produced. Plastic deformations are very small in a single forging simulation. Thus, the data need to be filtered to extract only those forces which cause plastic deformation. Such situations is recognized by analysing minimal displacements and material which is under deformation.

Tangent forces and forces parallel to workpiece flow direction are the most important factors in crack closing. This direction is obtained by analysing die and workpiece mesh from the two simulation steps. The distance difference for a forging node, which is the closest to analysed die node or surface, is considered. Then direction of material flow is the direction in which forces are analysed.

\section{Hybrid model}

The main idea of a hybrid model is usage of different dedicated models to simulate different wear mechanisms and combining their results in a single wear value. This approach requires not only computation of wear using different models, but also identification of coefficients of merging function, which in this research was a simple weighted sum function defined as:

$$
W_{\text {total }}=g_{\text {abrasive }} W_{\text {abrasive }}+g_{\text {adhesive }} W_{\text {adhesive }}+g_{\text {plastic }} W_{\text {plastic. }}
$$

where $g_{i}$ are weight coefficients and $W_{i}$ are results from different wear models. This merging function is simple, but it requires identification of weights. It is possible to use prior knowledge or analyse the case itself, e.g. by using an expert system. Results of this system are combined with an analysis of the die geometry. A simple processing routine has been developed to identify die areas, in which plastic deformation is likely to occur. Impact of this deformation is determined by analysing stresses acting on the die and small deformations which are repeated in subsequent forgings. Small deformation is used as a vector, which forms a line in die cross-cut. The line goes through a die volume until the surface (usually outside face of the die, if not, sum of other vectors is used) is inversely proportional to possible plastic deformation. The idea is based on the fact that if less material forms a protruding part exposed to high stresses, the more likely it will be affected by plastic deformation. The resulting lengths are normalized to form a coefficient for impact of the plastic deformation. Examples of measurements in two points in which impact of plastic deformation is different are shown in figure 2.

The system allows to use a case-based expert system to recognize wear mechanisms. In this type of system, die geometry is segmented into rules in which different wear mechanisms apply. It requires prior knowledge and exact identification of parameters in large number of rules, what is usually obtained from empirical data. The lesser data is in the system rules, the lower the quality of mechanisms identification will be. To combine rules, probability-based fuzzy approach was used in [16]. Such an attempt of classification by dividing typical geometry elements requires empirical data, which is not always accessible. The flowchart of hybrid model concept is shown in figure 3. 


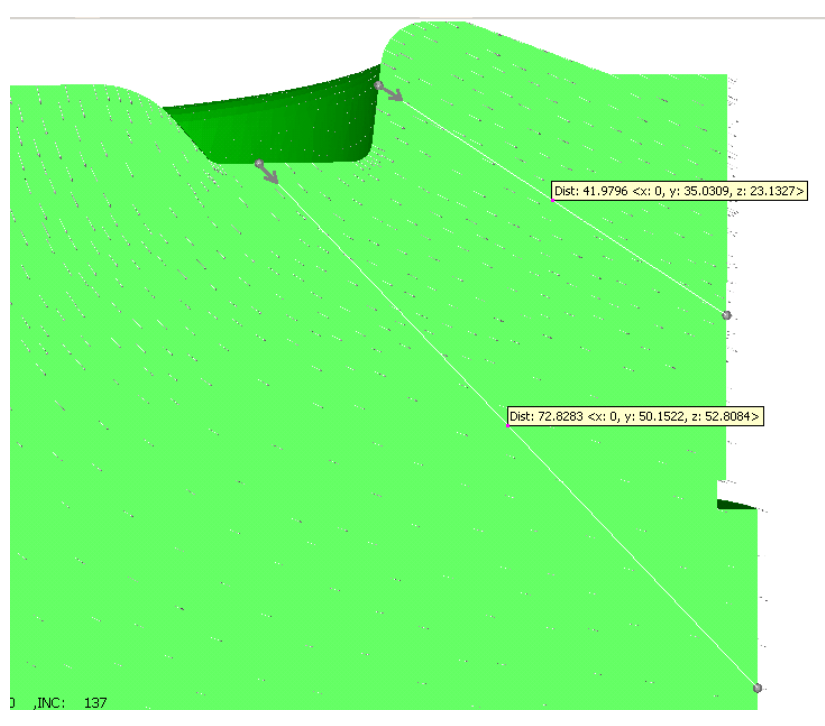

Figure 2. Measuring of lengths corresponding to amount of material prone to plastic deformation.

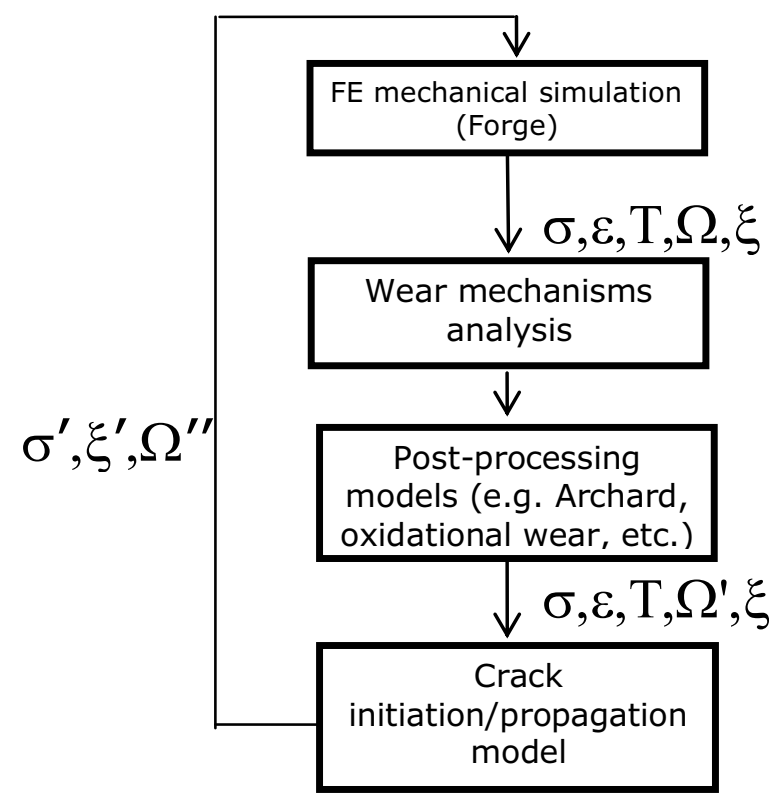

Figure 3. Data flow in hybrid wear model based on numerical simulation.

Work of the system begins with the FE simulation of a single process. Then, results are analysed and postprocessing models such as Archard model are applied. Geometry is analysed to determine importance of wear mechanisms. Some models, such as multi-scale approach to prediction of propagation and initiation of cracks, have to be started in the next stage, when other mechanisms of wear are applied on geometry. Then, wear is computed and the procedure is optionally restarted. It should be emphasized, however, that restarting the procedure should be avoided and prediction based on a single computations should be used to minimize the computing costs.
To verify hybrid model concept, simulations of the industrial forging were performed. Lower die in forging of a front wheel of the clutch described in [17] was considered. Nine measurement points, in which different wear mechanisms occur, were selected in this die, see. figure 4. Measurements of the die wear in this process were available.

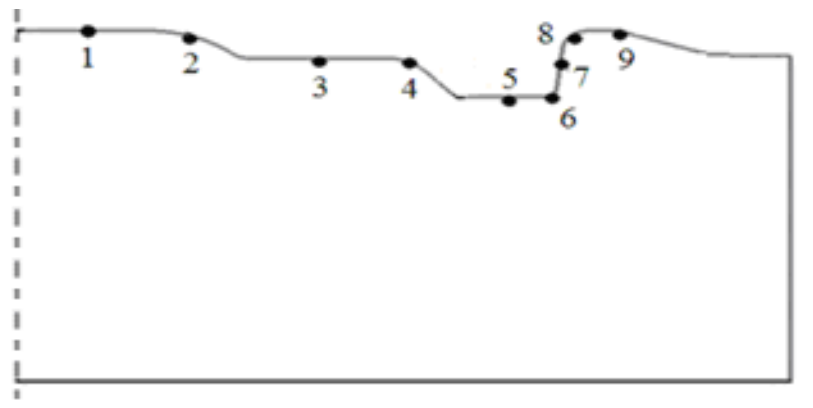

Figure 4. Analysed lower die with measurement points.

Simulation was a 3D FE and it included the die, which allowed to perform geometry analysis. The die material is $\mathrm{X} 40 \mathrm{Cr} 13$ steel with hardness of $52 \mathrm{HRC}$ after thermal processing. In the industrial forging cycle the wear was measured after 500, 6900 and 9000 forgings. Numerical computations of the abrasive wear have been performed. Following this parameters of the abrasive wear model have been identified as shown in [7]. Afterwards, plastic deformation model was computed and its impact was added giving a simple hybrid approach. Coefficients for equation (2) for two wear mechanisms have been identified by using mean square root error and geometry analysis. Values of plastic deformations at the die cross section are shown in figure 5.

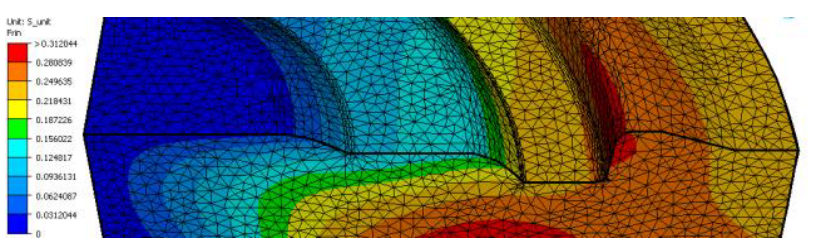

Figure 5. Visualization of die deformation during forging in a cross-cut plane.

Result of identification of equation (2) parameters is: $g_{\text {abrasive }}=0.999535, g_{\text {plastic }}=0.000465$. The results of comparison of measured and calculated abrasive wear for 9000 forgings are shown in figure 6. In points 1-4 the difference between hybrid and abrasive wear is relatively small, since plastic deformation is small. Calculated wear in points 5 and 6 is closer to measurements, but the plastic deformation in these points is probably caused mostly by force near point 8 , giving a "lever effect" on the die. It should be noticed that wear in point 8 is closer to measured values. Then, the geometry-based coefficients adaptation has been performed using method described in Chapter 4. The wear obtained from this adaptation is also shown in figure 6 and it is different in each point. It should be noticed that these results are closer to measured values even in points 4,5 and 6 . 


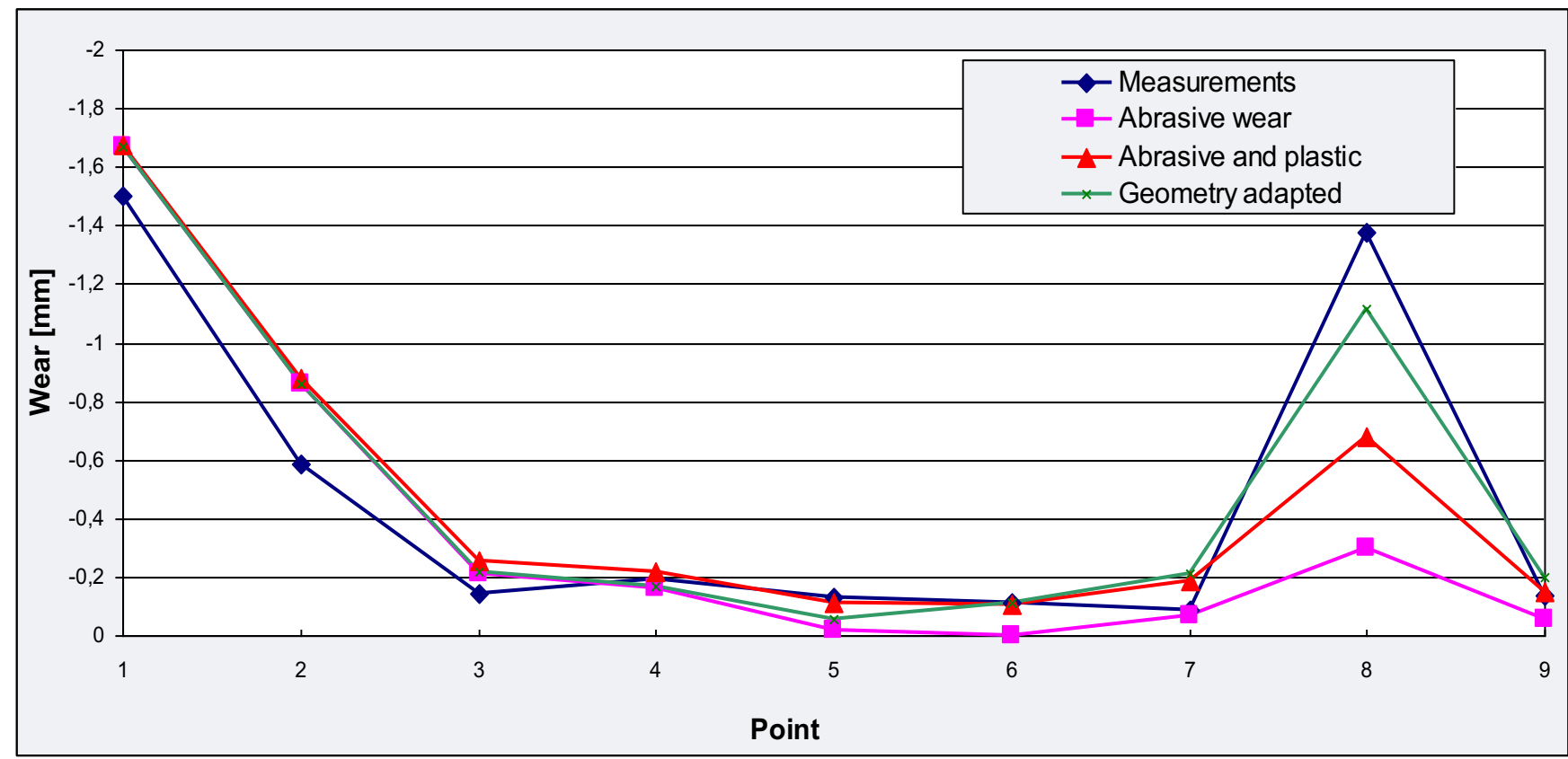

Figure 6. Results of abrasive and plastic wear computations with coefficient adaptation based on error and geometry

The values of computed coefficients are shown in Figure 7. It can be seen that in points 7, 8 and 9 plastic deformation have the biggest impact on wear, so the coefficient will be much higher in these points. In point 9, plastic deformation is mostly caused by material flash flowing outside a die, when deformed in the last stage of forging.

It is seen in figure 6 that application of the adaptation based on the error and the geometry resulted in a noticeable improvement of the accuracy of the model prediction in the point 8 comparing to the work [7]. The difference between measurements and predictions in other points than 8 are small and they may be a result of assumption that plastic deformation occurs uniformly in each of 9000 forgings.

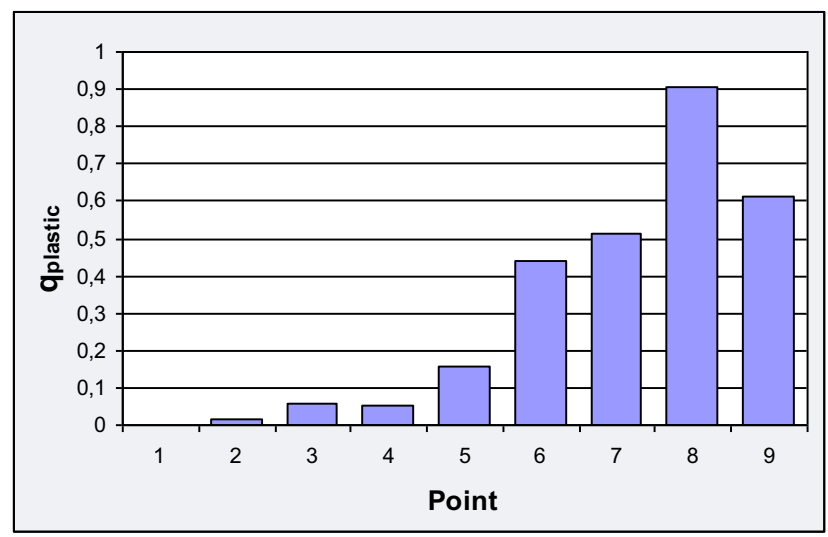

Figure 7. Values of plastic displacement wear coefficients at selected points.

General assumption, that plastic deformation occurs in a similar way in each cycle, is questionable. Exact computing of such deformations accounting for the die material rheology would require many cycles of simulations or metamodel trained from data acquired from real or simulated forging cycle. Part of the die with point 8 was indicated by geometry analysis as prone to plastic deformation (protruding part on workpiece expansion direction). The weight coefficient is relatively high, but computed values are still lower than measured.

\section{Conclusions}

It was shown in the paper that it is possible to combine different wear models to form a complex hybrid model, which covers different mechanisms of die destruction. In such approach even simple joining functions like weighted sum of components are sufficient. It is important to properly identify both parameters for different models of wear mechanisms, as well as coefficients for joining function. Analysis of results of simulations allowed evaluation of importance of different wear mechanisms in specified parts of the die. Wear mechanisms could be distinguished. These coefficients can also be computed using an expert system, which detects similarities in simulation results or parameters, but it requires empirical knowledge to be used as a case base.

It was shown in the paper that hybrid model approach is an efficient solution to combined wear mechanisms modelling. Further research would require an extension of the hybrid by including more single mechanisms models. More measurements of forging tools, including oxidation layers, crack parameters and surface properties, would be needed to reach this goal. Factors responsible for the impact of other wear mechanisms in parts of the die would have to be identified.

\section{Acknowledgements}

Financial support of the Polish National Science Foundation NCN, project, no. $2014 / 15 / \mathrm{B} / \mathrm{ST} 8 / 00187$, is acknowledged. 


\section{References}

1 B.-A. Behrens, F. Schäfer, F., Steel Res. Int. 80 (2009) 887-891.

2. S. Abachi, M. Akkok, M.I. Gokler, Tribology International 43 (2010) 467-473.

3. Y.-J. Kim, C.-H. Choi, Int. J. Precision Eng. Manuf., 10 (2009) 105-113..

4. Z. Gronostajski, M. Kaszuba, M. Hawryluk, M. Zwierzchowski, Arch. Civil. Mech., Eng. 14 (2014) 528-539.

5. J.F. Archard, J. Appl. Phys. 24 (1953) 981-988.

6. M.C. Shaw, Wear 43 (1977) 263-266.

7. M. Wilkus, S. Polak, Z. Gronostajski, M. Kaszuba, Ł. Rauch, M. Pietrzyk, Comp. Meth. Mater. Sci. 15 (2015) 311-321.

8. J.H. Kang, I.W Park, J.S. Jae, S.S. Kang, J. Mat. Proc. Techn. 941999 183-188.

9. C. Dahl, V.H. Vazquez, T. Altan, Proc. XX Conf. SENAFOR, Porto Allegre, 2000.

10. P.C. Paris M.P. Gomez, Anderson W.E., The Trends in Engineering, 13, 1961 9-14.

11. B. Tomkins, Philosophical Magazine 18 (1968) 1041-1066

12. M.M. Mirsayar, Materials and Design 86 (2015) 941-947.

13. J. Schroeder, D. Balzani, D. Brands, Arch. Appl. Mech. 81 (2011) 975-997.

14. J.L. Chaboche, European J. of Mechanics 13 (1994) 501-518.

15. C. Choi, A. Groseclose, T. Altan, J. Mat. Proc. Techn. 212, 2012, 1742-1752.

16. Z. Gronostajski, M. Hawryluk, M. Kaszuba, M. Marciniak, A. Niechajowicz, S. Polak, M. Zwierzchwoski, A. Adrian, B. Mrzygłód, J. Durak, Int. J. Adv. Manuf. Techn. 82 (2016) 1973-1991.

17. Z. Gronostajski, T. Będza, M. Kaszuba, M. Marciniak, S. Polak, Obróbka Plastyczna 25 (2014) 301-315. 